Meta

Journal des traducteurs

Translators' Journal

\title{
PAO et terminographie
}

\section{Caroline De Schaetzen}

Volume 36, numéro 1, mars 1991

La terminologie dans le monde : orientations et recherches

URI : https://id.erudit.org/iderudit/004038ar

Aller au sommaire du numéro

Éditeur(s)

Les Presses de l'Université de Montréal

ISSN

0026-0452 (imprimé)

Découvrir la revue

Citer cet article

De Schaetzen, C. (1991). PAO et terminographie. Meta, 36(1), 192-200.

Ce document est protégé par la loi sur le droit d'auteur. L'utilisation des services d'Érudit (y compris la reproduction) est assujettie à sa politique d'utilisation que vous pouvez consulter en ligne.

https://apropos.erudit.org/fr/usagers/politique-dutilisation/
Cet article est diffusé et préservé par Érudit.

Érudit est un consortium interuniversitaire sans but lucratif composé de l’Université de Montréal, l'Université Laval et l'Université du Québec à Montréal. Il a pour mission la promotion et la valorisation de la recherche. https://www.erudit.org/fr/ 


\title{
PAO ET TERMINOGRAPHIE
}

\author{
Caroline De Schaetzen \\ Institut Libre Marie Haps, Bruxelles, Belgique
}

Si elle n'est pas une panacée, la PAO s'indique dans les cas où le produit du travail terminographique est destiné à être publié ou — un luxe désormais à la portée des terminographes — si l'édition est requise pour faciliter la lecture du vocabulaire (enseignement, etc.) ou encore si le métalangage typographique permet une économie appréciable de rubriques et partant, de papier...

Le présent article décrit les fonctions de PAO utilisées dans l'édition de vocabulaires et rappelle quelques règles de typographie, de mise en page et de gestion en usage en lexicographie. Il se limite à la PAO textuelle : l'utilisation de l'infographie pour la préparation des dictionnaires spécialisés mérite un article à elle seule. Pour simplifier les choses, nous utiliserons ici le mot dictionnaire dans le sens générique de terminologie; il incluera donc les lexiques, vocabulaires, glossaires, nomenclatures, sauf si l'édition de l'un de ces ouvrages présente des particularités.

\section{TYPOGRAPHIE}

Les programmes de PAO permettent la définition d'une série d'attributs typographiques pour les caractères d'un document:

- les styles ou formes

- la famille (ou groupe de polices) et la police ou dessin des caractères

- le corps ou force de corps

- la graisse (intensité de l'encrage)

\section{POLICES ET CORPS}

Les mélanges de polices sont rares en lexicographie, où c'est le TIMES qui apparaît le plus souvent (il se lit plus vite, car c'est le caractère le plus fréquemment utilisé dans l'édition). L'absence d'empattement faisant plus «publicité», les vocabulaires techniques destinés au grand public peuvent par contre être imprimés en HELVETICA, par exemple. Les déformations des caractères sont à déconseiller pour les dictionnaires : elles cassent les proportions de la police et ralentissent ainsi la lecture; elles s'éloignent en outre des traditions typographiques de la lexicographie.

Le corps de la préface, des remerciements, de l'avant-propos, de l'introduction, du guide d'utilisation, de la table des matières d'un dictionnaire est souvent plus fort que celui du vocabulaire proprement dit; celui des index et de la bibliographie est généralement faible. Le corps des articles de dictionnaires est souvent de 6 ou 7 , l'échappement, d'une quarantaine de signes.

Dans les dictionnaires, les petites capitales sont fréquemment utilisées, en combinaison avec les grasses, pour les vedettes, parce qu'elles sont plus lisibles que les grandes capitales et qu'elles se marient mieux avec les caractères en bas de casse. Les termes apparentés, en fin d'article de dictionnaire, et les synonymes sont souvent en petites capitales (sans graisse). De plus, la première lettre de la vedette est souvent en capitales. On peut éventuellement recourir aux grandes capitales pour les vedettes, à condition de prendre pour elles un corps inférieur de un ou de deux points à celui de 
l'article. Dans l'article, les grasses servent au renvoi aux termes apparentés, aux expressions dérivées, aux formes fléchies, soit encore à la numérotation des différentes significations, en cas de polysémie. Il est donc utilisé pour les mêmes fonctions que les petites capitales.

L'emploi des italiques doit être cohérent; cela n'empêche pas ce style d'avoir plusieurs utilisations, désormais classiques, dans les dictionnaires : attestations (contextes, dans le vocabulaire de la terminologie), domaines d'emploi du mot, catégorie grammaticale (en abrégé), indicatif de niveau de langue (en abrégé également). Notons que trop peu de dictionnaires spécialisés utilisent des italiques.

CODES

L'utilisateur des logiciels d'éditique peut ajouter des codes à son texte.

En lexicographie, le losange noir est beaucoup utilisé parce qu'il prend peu de place. Il peut par exemple précéder un numéro en chiffres arabes, pour faire apparaître immédiatement une polysémie au lecteur du dictionnaire. Même chose pour le triangle noir renversé, qui peut précéder les synonymes ou les renvois aux termes apparentés. En terminologie, on pourrait gagner de la place en imitant les dictionnaires Robert, dans lesquels deux barres verticales (et non un retour-ligne) annoncent un antonyme.

Le tilde indique toujours la vedette, pour éviter sa recopie dans les expressions dérivées (notamment plurilexématiques) et les expressions fléchies reprises à l'intérieur de l'article. J. Rey-Debove (1971) rappelle qu'il vaut mieux ne pas utiliser de tilde dans l'index: l'index permuté simple est préférable (pour éviter une entrée d'index comme: non gardé, niveau, et un tilde après passage à).

En lexicographie, les crochets verticaux entourent toujours l'étymologie des mots ou expressions ou bien la transcription de leur prononciation, laquelle suit généralement la vedette. En terminologie, dit S. Tackels (1988), ils peuvent également indiquer une intervention extérieure dans une citation (suppression ou ajouts de mots).

Pour faciliter la frappe des caractères spéciaux fréquemment utilisés (tel le losange noir, pour les dictionnaires), on adopte généralement pour eux un code mnémonique, par macrocommande ou par recours à la fonction «glossaire» du traitement de texte. Signalons que le logo du copyright (un $c$ cerclé) est disponible dans la police ZAPF DINGBATS des imprimantes à laser POSTSCRIPT; la troisième de couverture ne doit donc pas nécessairement être composée par l'éditeur.

Il manque, dans le code ASCII des micro-ordinateurs, une série de symboles utiles pour la publication des dictionnaires techniques, tels ceux qu'avait conçus Wüster (exprimant les liens logiques entre les notions, par exemple) pour les vocabulaires de l'ISO.

La frappe simultanée des caractères autres que français, anglais, allemands, néerlandais, espagnols, italiens, portugais est difficile: elle requiert la frappe du code ASCII correspondant. Heureusement, certains logiciels de PAO sont multilingues : ceux de la société TEK et COMPULEXIS permettent l'écriture dans toutes les langues.

De nombreux éditeurs de dictionnaires de langue utilisent les chiffres arabes pour sous-catégoriser les grandes classes de significations, elles-mêmes distinguées par les chiffres romains. Dans certains dictionnaires techniques comme le dictionnaire commercial de Servotte, les expressions dérivées sont numérotées séquentiellement (mention inutile, qui alourdit un peu la présentation). Dans certains vocabulaires de l'Office de la langue française du gouvernement du Québec, ce sont les vedettes qui sont numérotées. 


\section{COMPOSITION}

La micro-édition permet également de composer le texte, c'est-à-dire d'en disposer les paragraphes et les pages.

La principale règle de composition applicable en terminologie est celle qui prévaut pour tous les imprimés : la conception sera réussie si elle est transparente au lecteur, si la présentation est fonctionnelle (l'habillage et les styles ne servent qu'à structurer le contenu du texte) et si elle est discrète (pas trop de variations et d'habillage), pour que l'attention du lecteur reste focalisée sur la communication, non sur la manière dont celleci est présentée. Ce précepte est particulièrement impératif pour les dictionnaires, qui sont des ouvrages dont la consultation doit être rapide.

\section{COMPOSITION EN PLACARDS}

\section{Fins de lignes}

La composition en placards est la gestion des fins de ligne.

Pour l'édition de dictionnaires, le programme de césures doit être fiable, vu l'étroitesse des colonnes de texte que comportent généralement ces ouvrages. Rappelons qu'en français, la césure doit respecter la division syllabique (en respectant les racines étymologiques), qu'elle ne peut intervenir dans un mot de moins de trois syllabes ni rejeter une syllabe muette en début de ligne ou dans un chiffre; on omet, enfin, de faire plus de trois césures successives et de couper le dernier mot d'un paragraphe. Les logiciels d'éditique permettent les césures dans plusieurs langues simultanément, indispensables pour les dictionnaires multilingues. Ces programmes ne sont pas exempts de défauts: l'algorithme de VENTURA génère un nombre élevé de coupures et fait des fautes (di-sposer, ada-ptation, inju-stice, rési-stance, a-mour, é-tat, o-pinion, ry-thme); pour parer à ces erreurs, on peut ajouter les mots souhaités dans un fichier spécial en y indiquant les césures permises ou bien ajouter une césure optionnelle dans les mots longs lors de la frappe du dictionnaire.

Peu de logiciels de PAO prévoient la justification verticale, indispensable pour les dictionnaires.

Les dictionnaires sont le plus souvent composés en fer à gauche lorsqu'ils comportent plusieurs colonnes (souvent deux colonnes de $6 \mathrm{~cm}$ environ, séparées par une ligne verticale), mais ils peuvent également être justifiés.

\section{Espacements et largeurs de ligne} de texte.

La composition en placards inclut la gestion des espacements ou largeurs des lignes

Même si on laisse le programme jouer sur l'espace intermot en lui donnant l'espace minimal, maximal et optimal, les modifications d'interlettrage restent de règle pour les dictionnaires, parce qu'elles sont élégantes si elles sont légères (afin de préserver la lisibilité du mot).

Dans les dictionnaires justifiés, on évite les blancs insécables, qui accroissent par compensation la taille des autres blancs de la ligne. Les titres (dictionnaires systématiques) ne sont jamais justifiés et sont toujours crénés. Les dictionnaires bicolonnes justifiés montrent généralement des variations dans l'interlettrage.

\section{Interlignage}

Dernière partie de la composition en placards, la gestion de l'interlignage (distance entre les lignes).

L'interlignage, la force de corps et l'espacement sont choisis de concert: on dira d'un texte dont le corps est 10 et l'interlignage de 12 points qu'il est composé en 10 sur 11. Le calibrage du texte ou calcul du nombre de pages doit être fait pour les différentes 
options possibles (une modification d'interligne ou de force de corps a des répercussions très importantes sur le volume de l'ouvrage). L'interlignage est souvent supérieur de deux points au corps mais moins le corps est fort, plus l'interligne peut être diminué.

Comme le corps des articles de dictionnaires est souvent de 6 ou de 7, 1'interligne est dès lors de 9 ou de 8 . L'interlignage de certains glossaires est double, pour guider l'wil dans l'attribution des équivalents aux termes en langue source. Dans les vocabulaires comportant des définitions pour les seuls termes difficiles, l'interlignage est souvent simple pour les différentes lignes des définitions. C'est le cas dans le vocabulaire de L. Boudreault Lapointe (1988) et autres Bulletins de terminologie du Secrétariat d'État du Gouvernement canadien.

Le passage aux mots commençant par une lettre de l'alphabet à ceux dont l'initiale est la lettre suivante doit être marqué par un nombre de lignes blanches suffisant, même s'il est accompagné d'une lettrine et/ou d'un filet horizontal gras (plus un bloc de texte représente une rupture avec ce qui précède, plus l'espace qui l'en sépare doit être grand).

\section{COMPOSITION PAR ZONES}

La composition par zones agence les différents paragraphes; l'ensemble des zones doit être présenté agréablement sur chaque page.

Dans les dictionnaires, la vedette est assez souvent en retrait (de 3 caractères au plus). Certains dictionnaires techniques sont au contraire en sommaire.

\section{COMPOSITION PAR PAGES}

En éditique, la composition par pages inclut la gestion des pages et des chapitres ou d'autres parties d'un livre.

Les marges sont déterminantes pour l'équilibre visuel d'une page de dictionnaire. Elles ne doivent pas être trop petites $(1 \mathrm{~cm}$ minimum et $1,5 \mathrm{~cm}$ pour les marges de reliure), pour permettre la lecture sans que les doigts du lecteur ne cachent le texte et pour laisser de la place à la reliure. Quant à la marge entre les colonnes, l'éditeur de dictionnaire lui donne, comme ceux qui publient d'autres ouvrages, une taille supérieure ou égale à deux fois la largeur maximale de la lettre $m$ du corps du texte (elle sera légèrement accrue si la colonne comporte un filet vertical).

Le nombre d'or, c'est-à-dire le rapport le plus courant entre la hauteur et la largeur d'un ouvrage est souvent de 1,4 (hauteur supérieure de $40 \%$ à la largeur). Toute variation importante par rapport aux normes (un format à l'italienne, c'est-à-dire horizontal, plutôt que vertical ou à la française; un rapport hauteur/largeur très différent du nombre d'or) entraînera une originalité très grande de l'ouvrage. Dans certains cas, cet écart de présentation est contre-indiqué (gros dictionnaires très techniques).

La présentation multi-colonne est essentielle à une lecture rapide des dictionnaires. Dans le cas des glossaires, l'entrée est dans la colonne de gauche, l'équivalent en langue étrangère, dans celle de droite. Dans les dictionnaires comportant des articles détaillés, l'équivalent se trouve dans l'article et les deux colonnes se lisent donc l'une à la suite de l'autre.

Comme dans tous les livres, les titres courants des dictionnaires sont toujours alignés sur les marges supérieures de gauche et de droite. Ils se composent toujours de la première et, pour le titre de droite, de la dernière entrée de la page. Le folio (numéro de page) peut ou non figurer dans un en-tête ou un pied de page; s'il est repris, il figurera soit dans la marge extérieure soit dans la marge intérieure (du côté de la reliure). Dans les gros dictionnaires, l'insertion des en-têtes ne peut être automatisée que par une macrocommande-programme du logiciel de traitement de texte. 
Le calibrage des illustrations ne pose pas beaucoup de problème en lexicographie, où les photos et les schémas sont beaucoup plus rares que dans les encyclopédies. Lorsqu'il y a plusieurs illustrations sur la même page d'un dictionnaire, on évite de les placer côte à côte.

\section{INDEX ET SOMMAIRES}

Comme les logiciels de traitement de texte, ceux de publication assistée permettent la génération automatique de glossaires et d'index.

La génération automatique d'un sommaire se justifie si l'ouvrage présente les notions par ordre systématique ou comporte une introduction et des commentaires circonstanciés (analyse linguistique du vocabulaire, bibliographie détaillée, etc.); dans ce cas, le foliotage est bien sûr indispensable, de même que la numérotation automatique des paragraphes de l'introduction et des commentaires.

Beaucoup de logiciels trient mal. Ils classent par exemple d'abord slide-key puis slide-way et enfin slideway et non slide-key, slideway et slide-way; ils suivent en outre l'ordre suivant: 0,2 limit, 1000 years, 2-hand control puis left-hand rotation au lieu de: 0,2 limit, 2-hand control, 1000 years et left-hand rotation (Felber 1985).

Les index sont généralement bien composés par les logiciels de traitement de texte ou d'éditique (mise en retrait de la seconde ligne d'une entrée d'index dépassant une ligne, virgule et espace entre l'entrée et le numéro de page). Ils peuvent être utilisés pour inverser le dictionnaire, si celui-ci est paginé. Si, dans l'index, le renvoi est par contre fait à la vedette, l'index devra être généré par un programme écrit dans le logiciel de traitement de texte avec lequel le dictionnaire a été rédigé.

\section{HABILLAGE}

L'habillage est l'ensemble des «décorations» qui peuvent être apportées aux documents.

Dans les lexiques de vulgarisation du Secrétariat d'État du Canada, un filet sépare parfois les entrées commençant par la même lettre de l'alphabet de celles qui commencent par la lettre précédente et suivante.

Les changements de lettres sont souvent annoncés par une lettrine habillée sur deux ou trois lignes (ou bien par un titre de chapitre composé de la nouvelle lettre, en corps fort et en grasses). Ces lettrines figurent toujours soit sur une belle page (recto du feuillet) soit sur une fausse page (verso).

Le document sera généralement photocomposé en monochromie (en une couleur, c'est-à-dire en deux couleurs, puisque le noir ne «compte pas»), en bi- ou quadrichromie (trois ou cinq couleurs). La couleur étant extrêmement onéreuse, les caractères des dictionnaires sont généralement noirs sur fond blanc. La bichromie est rare: dans le Micro-Robert, les vedettes sont en rouge, ce qui les fait ressortir et donne une lisibilité plus grande à l'ouvrage, destiné aux enfants d'âge scolaire. Dans les petits lexiques de vulgarisation de l'Office de la langue française, qui sont classés par ordre alphabétique strict (langues anglaise et française confondues), les termes anglais sont en bleu et les termes français sont en noir.

\section{AUTOMATISATION DE L'ÉDITION}

Une grande partie des fonctions d'édition décrites ci-dessus peuvent être demandées automatiquement à l'ordinateur.

Les commandes de mise en forme ou de codes d'habillage fréquemment utilisés peuvent être attribuées à une touche, notamment de fonction. 
Une composition et un habillage entiers peuvent être stockés par l'utilisateur dans un fichier appelé «feuille de style», et utilisés systématiquement pour le même type de texte. L'utilisateur choisit, parmi les feuilles offertes par les concepteurs de son logiciel, celle qui est la plus proche de la présentation globale de son ouvrage; il y apporte simplement quelques modifications et sauvegarde cette feuille modifiée sous un autre nom. Un centre de terminologie ou un terminographe indépendant peut ainsi créer une feuille de style pour les glossaires très techniques, une autre pour les glossaires de vulgarisation, dont la mise en page est généralement plus sophistiquée, et enfin une troisième pour les dictionnaires proprement dits. La conception de feuilles de style peut également être sous-traitée à une société comme SYNAPS pour VENTURA, par exemple.

Les premiers logiciels de composition permettaient déjà le groupement de commandes dans des fichiers ou macrocommandes. Une macro peut par exemple prévoir un espace, dont la taille sera demandée à l'utilisateur, pour l'insertion d'une figure dans une colonne de dictionnaire.

\section{PAO SPÉCIALISÉE}

\section{INTERFACES AVEC LES SGBD}

Il vaut mieux ne pas saisir les données d'un vocabulaire à publier en traitement de texte, mais sur un gestionnaire de fichiers au sens large (gestionnaire de bases de données à zones de taille variable, pour économiser de la mémoire de masse), même pour un dictionnaire de plus de 1000 termes comportant définitions, attestations et notes encyclopédiques et/ou linguistiques nombreuses. Beaucoup de fonctions de traitement de texte facilitant la frappe et la correction des entrées et des vedettes peuvent être programmées par macrocommande sur un gestionnaire comme RAPIDFILE.

VENTURA ajoute des fonctions de PAO à certains progiciels de bases de données, permettant d'éditer ces bases de manière normalisée et élégante. La société SYNAPS met par exemple automatiquement en page des informations stockées dans n'importe quelle base de données, comme un dictionnaire: les zones de la base sont annotées de certains caractères ASCII reconnus par la feuille de style VENTURA, qui les convertit en commandes de mise en page ou de typographie. Quant à ALDUS, elle développe actuellement une version de PAGEMAKER autour d'une base de données partageable. Les auteurs de dictionnaires travaillant avec des systèmes de gestion de bases de données peuvent également programmer l'impression d'un ouvrage en se servant d'un programme comme LATEX, qui met à leur disposition un grand nombre de styles: des routines écrites dans le SGBD en question peuvent associer ces styles aux différentes entrées du dictionnaire (l'italique aux exemples, etc.). Certains responsables de banques de terminologie publiant des lexiques sur base de leur fond disposent d'un système similaire, conçu pour eux (TEAM,BTQ).

\section{UN ÉDITEUR DE DICTIONNAIRES}

On l'a dit, il existe au moins un programme d'édition de dictionnaires sur microordinateurs, et qui est directement interfaçable avec des photocomposeuses: COMPULEXIS, qui, pour les lexicographes, est en même temps un gestionnaire de bases de données conçu pour les dictionnaires.

\section{Configuration requise}

Ce logiciel anglais tourne sur les AT et les PS/2 pourvus d'une disquette de 1,2 ou de 1,44 mégas au moins, d'un disque rigide d'au moins 30 mégas, d'un clavier étendu 
(101 touches), d'une carte EGA ou VGA et d'une version 3.1 ou postérieure du DOS. Un disque rigide de 30 mégas est recommandé.

Les imprimantes qu'il supporte sont la LASERJET de HEWLETT-PACKARD et compatibles, pourvues d'une mémoire RAM d'au moins 1 méga. L'interface avec POSTSCRIPT est prévue pour la fin de l'année 1991.

COMPULEXIS fournit lui-même les fontes requises parce qu'il leur a inclu les signes nécessaires pour la typographie des dictionnaires de toutes langues.

\section{Saisie}

Ce gestionnaire de fichiers à zones et enregistrements de taille variable permet la saisie, le stockage et la recherche des différents éléments d'une entrée de dictionnaire par le truchement d'annotations brèves d'un texte continu; ces tags indiquent le type d'information lexicographique (vedette, équivalent, information grammaticale, etc.) que constitue la chaîne de caractères annotée et permettent sa mise en forme typographique subséquente (par commande globale).

L'écran de saisie affiche des «boîtes» différentes pour chaque type de données de l'article. Pour les dictionnaires bilingues, l'écran esı divisé en deux parties pour la saisie et l'affichage.

Les séparateurs des différentes données de l'article (losange, point-virgule, saut de ligne, barres obliques ou crochets) peuvent être générés automatiquement par COMPULEXIS, lequel permet également de faire dépendre le traitement d'un type de données du contenu d'une zone ou même des types de données qui l'environnent. Les éléments de données fixes (utilisés par exemple dans les références croisées) peuvent également être générés, de même que les éléments hiérarchiques des différents articles.

L'utilisateur peut saisir des données cachées et donc stocker en marge des articles des renseignements qui ne peuvent pas être publiés, telles des notes, des réflexions diverses, l'étape en cours (deuxième correction d'épreuves, etc.).

\section{Présentation}

Bien que le texte soit introduit au kilomètre et dans la même police, le système interprète les données: il peut à tout moment les afficher et les imprimer sous la présentation qu'elles auront sur une page du dictionnaire imprimé. La typographie est donc un attribut lié au type de données et non une commande à introduire partout où elle est nécessaire.

Les données peuvent être copiées sous forme de fichier ASCII, sous un format utilisable pour d'autres applications.

\section{Manipulation} fichier.

Les différents types de données sont chânés en articles; ceux-ci sont chaînés en un

Les entrées sont indexées en permanence et automatiquement.

Des zones, des articles ou des portions d'articles peuvent être insérés, effacés, copiés, déplacés, séparés et fusionnés à tout moment; il est donc possible de dériver des dictionnaires de dictionnaires plus complets ou d'inverser un dictionnaire.

Une fonction de recherche permet le positionnement rapide sur un type de données ou le début d'une chaîne précise dans un article. Les recherches et remplacements globaux (conditionnels ou non) d'articles au sein de données précises ou n'importe où dans le dictionnaire sont possibles.

\section{Impression}

L'impression peut être sélective et porter sur un article, certains articles dont la vedette commence par une lettre faisant partie d'une portion précise de l'alphabet, sur tout un fichier ou bien sur certaines parties de l'article (la vedette seulement, par 
exemple), et ce pour tous les enregistrements ou certains d'entre eux. Des opérateurs booléens permettent de combiner les filtres décrits ci-dessus. Ces listages partiels permettent, au cours de l'élaboration d'un dictionnaire, de faire diverses vérifications (renvois croisés, vérification des définitions des entrées d'un sous-domaine par un expert, etc.).

Les concepteurs du logiciel ont conçu certaines polices pour les imprimantes compatibles pour faciliter l'impression des caractères et des signes diacritiques de toutes les langues, y compris ceux de l'alphabet phonétique international, ainsi que l'impression des symboles requis en lexicographie.

COMPULEXIS permet donc au lexicographe et au terminographe de se concentrer sur la partie noble de leur travail, au lieu de perdre du temps à vérifier la consistance de la ponctuation, de la typographie, de l'ordre alphabétique, etc.

\section{CONCLUSIONS}

\section{ON DEMANDE INTERFACES..}

Des interfaces de logiciels d'éditique avec les logiciels de bases de données sont attendues avec intérêt par les éditeurs de dictionnaires. Les logiciels de publication assistée sont conçus pour des textes de formats libres, non pour des fichiers mélangeant texte libre et fixe, comme les dictionnaires, catalogues et autres annuaires. Les auteurs de dictionnaires sont donc contraints de passer d'un logiciel à l'autre (traitement de texte, gestionnaire de bases de données, PAO). D'où l'intérêt de COMPULEXIS...

\section{DES DICTIONNAIRES MOINS BIEN COMPOSÉS?}

L'autorité de l'imprimé n'aura encore cours que si les imprimés restent rares. À cet égard, les frais d'impression étaient un bon filtre à la qualité, même si des ouvrages n'avaient pas l'auditoire qu'ils méritaient. Les facilités de la micro-édition risquent en effet d'être utilisées par des auteurs ou «éditeurs» peu scrupuleux pour masquer le caractère provisoire ou la portée locale de certains vocabulaires... Ce phénomène risque d'entraîner par ricochet le recours, par les éditeurs de dictionnaires de qualité, à des formes d'édition plus coûteuses, afin de démarquer leurs ouvrages des travaux moins bons.

On risque en outre d'assister, dans un premier temps, à un appauvrissement et à une standardisation de la présentation des terminologies, voire à la disparition d'une partie d'un métalangage typographique. Le manque de maîtrise des techniques d'édition, inévitablement lié à leur vulgarisation, risque ainsi de supprimer une partie des connotations attachées au papier, aux couleurs, à la reliure, tous éléments facilitant l'évaluation des dictionnaires et donc leur acquisition par le public cible de l'éditeur.

\section{DES DICTIONNAIRES PLUS BAVARDS?}

Au contraire, en manipulant les fonctions et logiciels d'édition assistée, les terminographes prendront peut-être conscience de la puissance du métalangage typographique et de celui de la composition; ils constateront les économies de place et la souplesse, donc la rapidité d'identification des informations, qu'ils permettent. Il se peut qu'alors, on trouve dans les dictionnaires de spécialités toute la palette des symboles qui parsèment les dictionnaires de langue ou le dictionnaire de la machine-outil de Wüster; ces codes remplaceraient avec bonheur une série de rubriques en clair qui sont scolaires et prennent de la place.

En maîtrisant eux-mêmes les paramètres de l'édition et en étant moins prisonniers des coûts de la publication, peut-être les terminographes ajouteront-ils également à leurs 
travaux un éventail d'informations plus large, analogue lui aussi à celui que présentent les dictionnaires de langue. En même temps que leur présentation est pauvre et rigide (à l'image de celle des banques de termes?), les ouvrages des grosses maisons d'édition de dictionnaires techniques ou des grands centres de terminologie sont peu loquaces sur les notions et les dénominations; aucun dictionnaire technique ne donne par exemple les informations suivantes, qui ne relèvent pourtant pas de la connaissance encyclopédique des notions:

- sous-domaine

- domaine d'application

- marques socio-linguistiques: niveau de langue (standard, soutenu, relâché); variantes professionnelles (jargon d'atelier; vocabulaire administratif; jargon de firme; communications commerciales; discours académique...); variantes textuelles (utilisé surtout dans les manuels d'utilisateur); variantes géographiques (importantes pour une série de néologismes français forgés au Canada et non diffusés en Europe); degré de diffusion (en voie de disparition, désuet).

Autres points de présentation des dictionnaires, qui ne font pas toujours l'objet d'une réflexion préalable :

- le classement des synonymes (ordre alphabétique ou selon leur fréquence?)

- $\quad 1$ 'ordre des rubriques: par défaut ou fixe?

- le point ou l'espace après une abréviation (indiquant le code grammatical, par exemple)? Insertion de barres obliques dans la chaîne?

— le métalangage : ou, etc., =, ", ', ()

- la consistance des références

- l'orthographe des sigles et des majuscules (patronymes).

\section{BIBLIOGRAPHIE}

«Aldus. Mettre l'avenir en pages» (1989): La semaine informatique, Bruxelles, 157, LSI.

«Desktop publishing ou l'édition électronique» (1989) : PC Micro Magazine, 68, Bruxelles, Ecopress.

BOUDREAULT LAPOINTE, L. (1988): Vocabulaire de biotechnologie végétale, Hull, Secrétariat d'État du Canada.

COMPULEXIS, description du logiciel (non publié).

GROUT, B. ATHANASOPOULOS, I. et R. KUTLIN (1986): Desktop publishing from A to Z, Berkeley, Osborne Mc Graw-Hill.

MERMET, F. et G. (1988): Mise en page avec VENTURA, Paris, Cédic Nathan.

REY-DEBOVE, J. (1971): Étude linguistique et sémiotique des dictionnaires français contemporains, The Hague-Paris, Mouton.

TACKELS, S. (1988): Typographie et terminologie. Guide de présentation des travaux terminologiques, coll. Études, recherches et documentation, Québec, Office de la langue française, Éditeur officiel du Québec. 